\title{
Trophoblast Biology, Biochemical Therapy and Prevention of Choriocarcinoma
}

\section{Kazuo Maeda*}

Department of Obstetrics and Gynecology, Tottori University Medical School, Japan

\begin{abstract}
Biochemical tumor marker was human chorionic gonadotropin (hCG) produced by choriocarcinoma itself, which was used to determine the complete remission of the carcinoma, which was treated by primary systemic methotrexate (MTX) therapy. Also, hCG was the marker of positive pregnancy test in the MTX prevention of choriocarcinoma. It will be important to use biochemical marker produced by the subject in the chemotherapy of malignant tumor.
\end{abstract}

Keywords: Malignant tumor; Choriocarcinoma; hCG; Complete remission; Prevention

\section{Introduction}

Trophoblast is very particular fetal origin cells of chorionic membrane of placental villi classified into syncitio trophoblast forming outside layer of the villi and cytotropholast, which covers inner layer of the villi, which forms chorionic membrane of the villi (Figures 1 and 2). They are very particular cells existing in the placenta during pregnancy, but physiologically do not exist in human after birth, while they play the important role to transfer fetal nourishing materials with active transfer function, and transfer oxygen to fetal blood from maternal arterial blood, and also transfer carbon dioxide $\left(\mathrm{CO}_{2}\right)$ from the fetal to maternal blood with passive transfer function in the placenta during pregnancy. As the placenta is expelled in the delivery process, they do not physiologically exist in the newborn after the birth.

Trophoblasts prepare hazardous function, e.g., ectopic pregnancy often develops tissue destruction and hemorrhage, develops placenta accreta destructing the myometrium during pregnancy. Heavy tissue destruction occurs in the choriocarcinoma composed of two kinds of trophoblasts (Figure 2). The placental site trophoblastic tumor (PSTT) is composed of intermediate trophoblast (Figure 3). Molar tissue develops destructive hydatidiform mole during molar pregnancy (Figure 4).

Trophoblasts prepare endocrinological function, i.e., it excrete human chorionic gonadotropin (hCG), which indicate the pregnancy by positive pregnancy test, and its high titer suggests trophoblastic diseases, including hydatidiform mole and choriocarcinoma, The dissemination of small number of trophoblasts in endometrium after hydatidiform mole made the pregnancy test positive (Figure 5), and a complete remission of choriocarcinoma due to effective methotrexate (MTX) chemotherapy was known by the disappearance of tumor cells (Figure 6) and hCG in the serum and urine, namely, the hCG is the most sensitive tumor marker of choriocarcinoma.

The excretion of steroid hormones, e.g., estrogen and progesterone develop body temperature rise, morning sickness, softening of birth canal or other pregnancy signs.

Biochemical nature of trophoblast is characteristic in the chemotherapy of trophoblastic disease, namely, MTX was the antimetabolite drug of choriocarcinoma, namely, trophoblasts needs folic acids to grow, where chemical structure of MTX was close to folic acid, therefore, choriocarcinoma absorbs MTX instead of folic acid, where MTX does not contribute the growth of choriocarcinoma, then choriocarcinoma was atrophic and then necrotized [1].
Thus, MTX chemotherapy [1] and prevention [2] of choriocarcinoma was related biochemical nature of trophoblasts.

\section{Methods and Results}

\section{Choriocarcinoma chemotherapy with MTX}

Choriocarcinoma had been treated primarily hysterectomy then chemotherapy of no MTX before 1960, where general metastasis was common, and the patient frequently died by the brain metastasis. Maeda recognized choriocarcinoma as systemic disease but not a local one, then changed its treatment to primary chemotherapy, which was the systemic chemotherapy without local therapy, but the treatment was started with choriocarcinoma sensitive anticancer agent, that was MTX associated with actinomycin $\mathrm{D}$, where cancer status was monitored by measuring direct biochemical tumor marker, which was the hCG of choriocarcinoma, where sudden increases of ultrasonic Doppler tumor flow wave resistance index and pulsatility index immediately after the start of chemotherapy, which was the sign of choriocarcinoma sensitivity to MTX. Continuous response of choriocarcinoma to MTX was studied by the reduction of metastatic tumor, serum $\beta$ hCG and urinary hCG. Complete remission was determined by the disappearance of uterine primary tumor and disappearance of tumor hCG, namely, direct tumor secretion was the marker of complete remission. Thereafter the patient had normal pregnancy in the uterus, that was the final sign of complete remission [1].

Repeated MTX therapy was Infusion of $0.5 \mathrm{mg} / \mathrm{kg} /$ day associated Actinomycin D $0.5 \mathrm{mg}$ in 3-4 days in a week, and repeated next week. MTX 20-25 mg/day infusion was carried out in a brain metastasis, who had complete remission for 20 years.

Side effects was dermatitis, alimentary tract erosion, hair fall, leucopenia, hepatic lesion, etc. Leucovorin, folic acid agent, was injected 2 hours after MTX infusion to prevent bone marrow damage.

*Corresponding author: Kazuo Maeda, Honorary Professor, Department of Obstetrics and Gynecology, Tottori University Medical School, 3-125 Nadamachi, Yonago, 683-0835, Japan, Tel: 81859226856; E-mail: Maedak@mocha.ocn.ne.jp Received November 21, 2016; Accepted December 03, 2016; Published December 08, 2016

Citation: Maeda K (2016) Trophoblast Biology, Biochemical Therapy and Prevention of Choriocarcinoma. Clin Med Biochem 2: 121. doi:10.4172/24712663.1000121

Copyright: () 2016 Maeda K. This is an open-access article distributed under the terms of the Creative Commons Attribution License, which permits unrestricted use, distribution, and reproduction in any medium, provided the original author and source are credited. 
Citation: Maeda K (2016) Trophoblast Biology, Biochemical Therapy and Prevention of Choriocarcinoma. Clin Med Biochem 2: 121. doi:10.4172/2471-2663.1000121

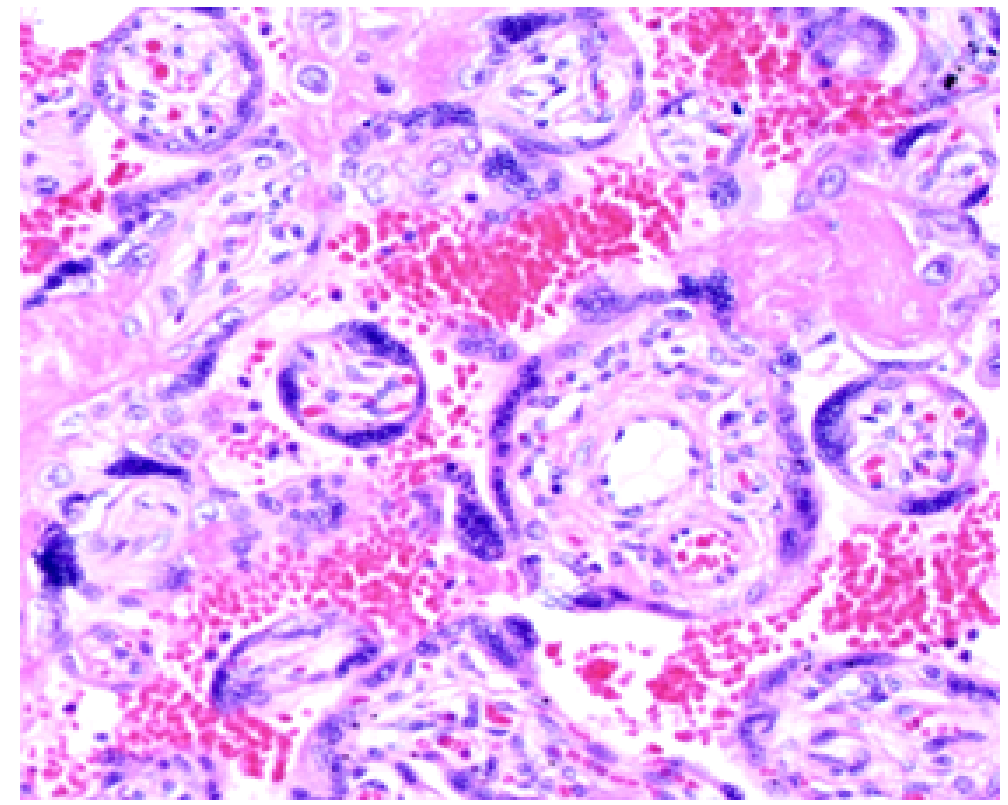

Figure 1: Placental villi covered by syncytiotrophoblasts outside and cytotrophoblasts inside in a fibrin deposit case.

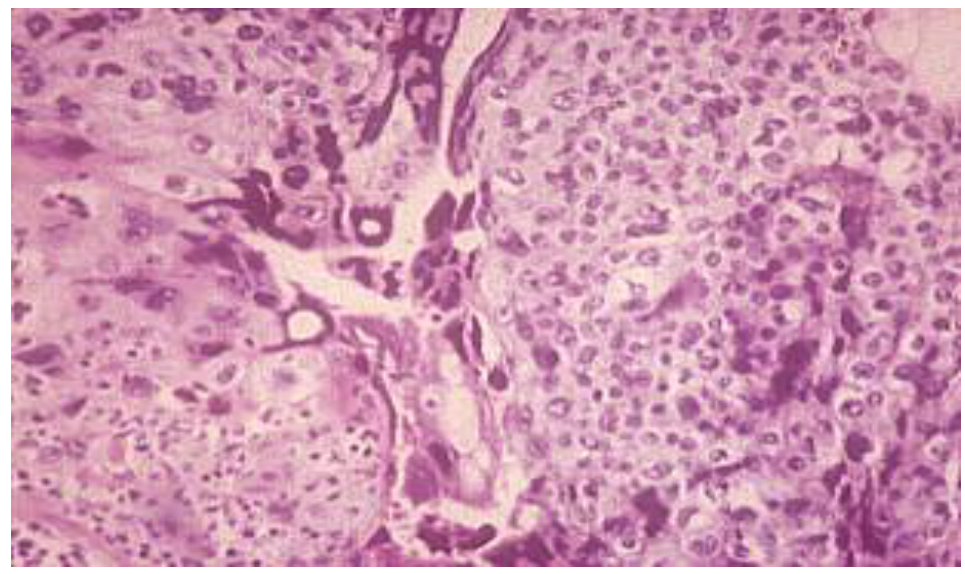

Figure 2: Syncytio- and cyto-trophoblasts in a choriocarcinoma. HE stain, 200X.

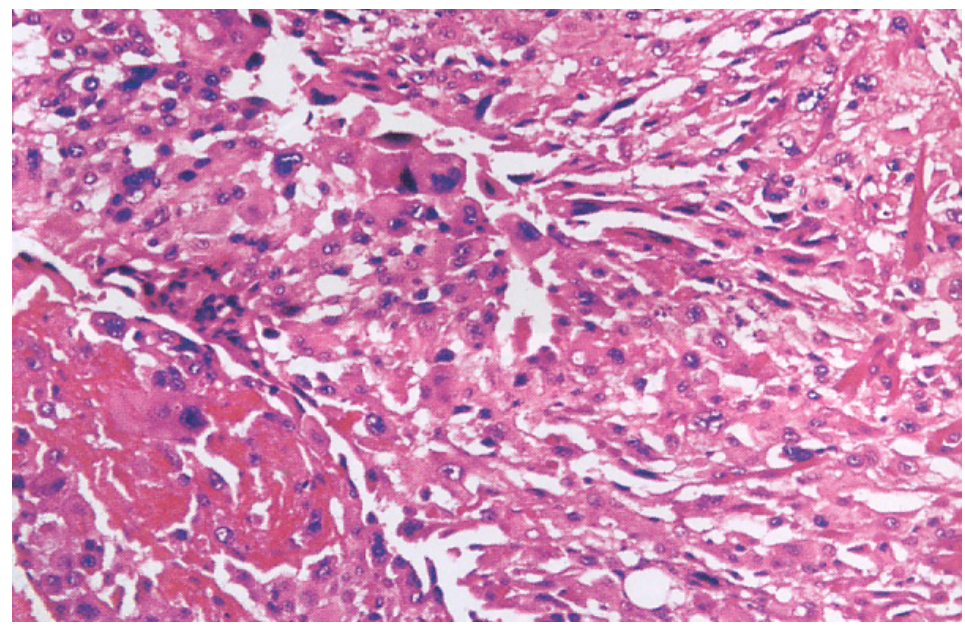

Figure 3: Intermediate trophoblasts of a PSTT (Courtesy Dr. U Honemeyer). 
Citation: Maeda K (2016) Trophoblast Biology, Biochemical Therapy and Prevention of Choriocarcinoma. Clin Med Biochem 2: 121. doi:10.4172/2471-2663.1000121

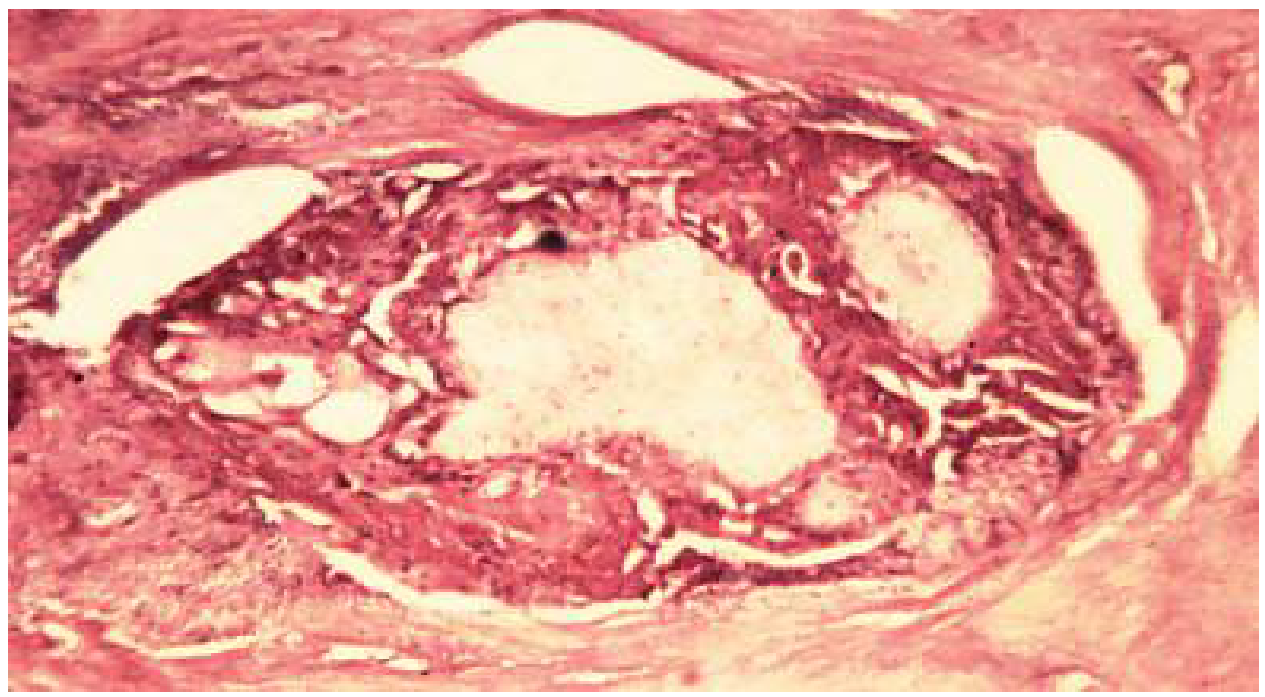

Figure 4: Microscopic view of destructive mole, which was a hydatidiforme mole invading myomtrium, where placental villi structure was preserved, while choriocarcinoma keeps no villi structure, namely, choriocarcinoma is immature but destructive mole is mature in the histology.

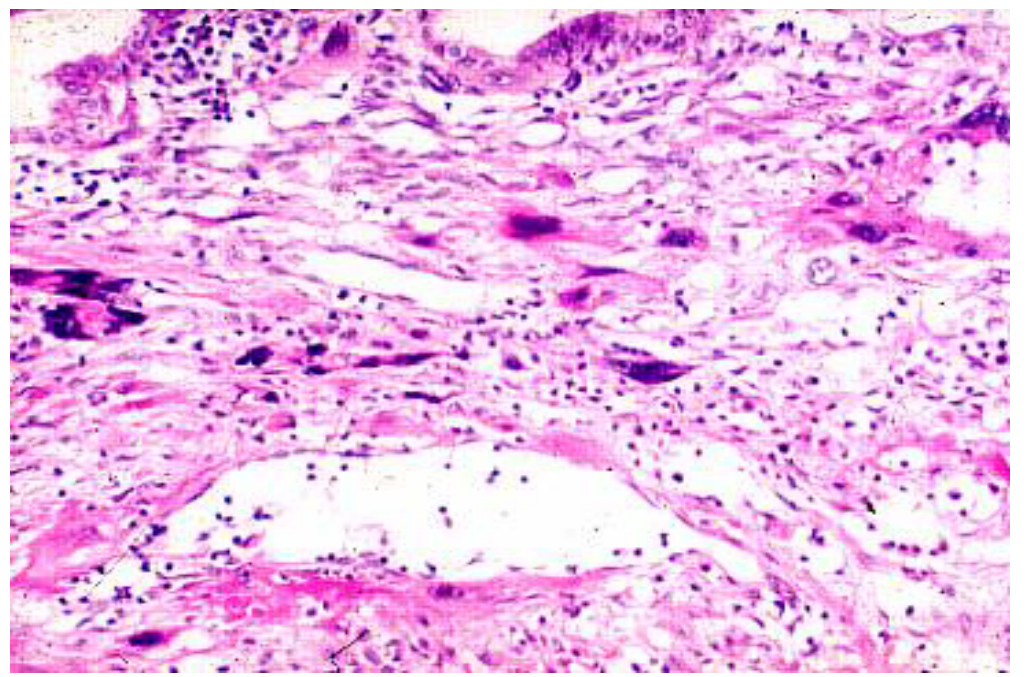

Figure 5: Syncytiotrophoblasts embeded in postmolar endometrium may cause prolonged positive pregnancy test, possively preceding choriocacinoma? HE stain, 200X.

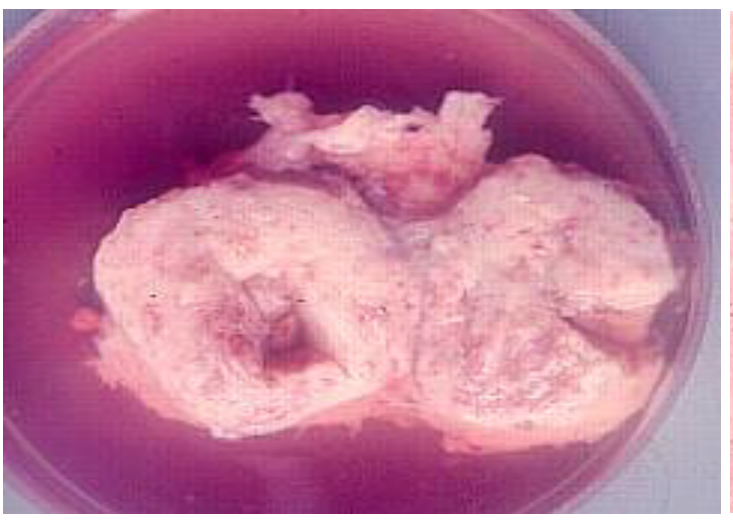

A. Necrotic uterine choriocarcinoma.

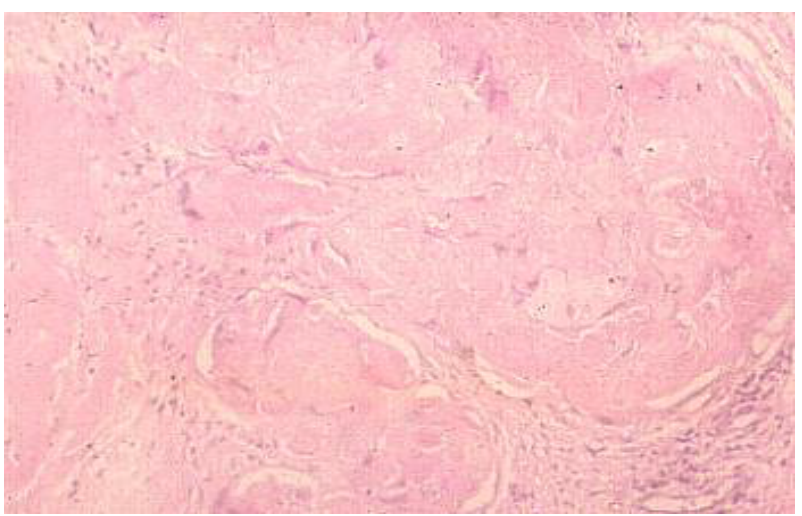

B. Microscopic view of necrotic tumor. HE, 200X.

Figure 6: Necrotic uterine choriocarcinoma after systemic MTX therapy. Compare microscopic view to Figure 2 choriocarcinoma before the chemotherapy. 
Citation: Maeda K (2016) Trophoblast Biology, Biochemical Therapy and Prevention of Choriocarcinoma. Clin Med Biochem 2: 121. doi:10.4172/2471-2663.1000121

A case received bone marrow transplantation in heavy leucopenia. Hepatic function was frequently studied.

\section{Choriocarcinoma prevention with MTX}

The reason of choriocarcinoma prevention: Choriocarcinoma frequently developed after the complete hydatidiform mole. Although there was post molar monitoring performed by frequent hospital visit, pregnancy test, basal body temperature and chest X-ray, after the hydatidiform mole, Maeda planned more active choriocarcinoma prevention with chemotherapy.

As there was trophoblastic infiltration in the endometrium after the evacuation of hydatidiform mole, there was concern to develop choriocarcinoma from the endometrial trophoblasts. There was concern to develop choriocarcinoma in the cases of continuously positive pregnancy tests after hydatidiform mole. It was possible to erase tumor trophoblasts by MTX in choriocarcinoma.

Therefore, we planned MTX therapy of 107 cases comparing to 87 no MTX cases after hydatidiform mole.

The methods of choriocarcinoma prevention: We compared 2 groups: one group was 107 cases who received oral MTX after evacuation of complete hydatidiform mole. They received daily $10 \mathrm{mg}$ oral MTX for 7 days. There were 2 cases of positive pregnancy test in the group, who received MTX until negative pregnancy test, where 200 and $300 \mathrm{mg}$ MTX was administered before negative pregnancy test.

According to the results, there was no choriocarcinoma in 107 cases who received MTX. Observation period was 2 years. Another group was 87 cases after hydatidiform mole who received no MTX, and developed choriocarcinoma in 6 cases (7\%) in the observation period.

The development of choriocarcinoma was significantly less in the group of MTX administration than no MTX group [2]. A randomized controlled trial (RCT) was performed by UICC (unio interntionalis contra cancerum) for the prevention of choriocarcinoma after our trial, where the results were the same as our study.

\section{Discussion}

MTX was antimetabolite of folic acid which was indispensable to choriocarcinoma, and the hCG, which was the tumor marker of choriocarcinoma, was the direct product of trophoblast, namely, biochemical condition was important in the complete remission of MTX chemotherapy of choriocarcinoma and its prevention. Therefore, any other biochemical condition will be carefully considered in the chemotherapy of common cancer.

Complete remission of choriocarcinoma achieved by chemotherapy was reported several times after our study [3-6], while no active prevention of choriocarcinoma with MTX chemotherapy has been found in recent reports after our study, though it is an important obligation of researchers for choriocarcinoma.

\section{Conclusion}

As the biochemical condition was recognized important in the complete remission and prevention of choriocarcinoma, biochemical condition should be considered also in the chemotherapy of common cancer.

\section{References}

1. Maeda K, Yoshioka S, Iba N (1970) Treatment of choriocarcinoma. Obstet Gynecol Ther 20: 483-492.

2. Koga K, Maeda K (1968) Prophylactic chemotherapy with amethopterin for prevention of choriocarcinoma following removal of hydatidiform mole. Am J Obstet Gynecol 100: 270-275.

3. Li J, Yang J, Liu P, Ren T, Zhao J, et al. (2016) Clinical characteristics and prognosis of 272 postterm choriocarcinoma patients at Peking Union Medical College Hospital: a retrospective cohort study. BMC Cancer 16: 347.

4. Han SN, Amant F, Leunen K, Devi UK, Neven P, et al. (2012) Treatment of highrisk gestational trophoblastic neoplasia with weekly high-dose methotrexateetoposide. Gynecol Oncol 127: 47-50.

5. Capdevila J, Maroto P, Sainz S, Villavicencio H (2006) Disseminated retroperitoneal choriocarcinoma with open fistula to intestine. "Restitutio ad integrum" with chemotherapy alone. Clin Transl Oncol 8: 453-455.

6. Hiramatsu Y, Masuyama H, Ishida M, Murakami K, Sakurai M (2005) Term delivery choriocarcinoma patient with brain and lung metastases successfully treated by etoposide, methotrexate, actomycin D, cyclophosphamide and vincristine (EMA-CO) chemotherapy. Acta Med Okayama 59: 235-238. 